\title{
Use of model predictive control and weather forecasts for energy efficient building climate control
}

\author{
Frauke Oldewurtel ${ }^{\mathrm{a}, *}$, Alessandra Parisio ${ }^{\mathrm{b}}$, Colin N. Jones ${ }^{\mathrm{c}}$, Dimitrios Gyalistras ${ }^{\mathrm{a}}$, \\ Markus Gwerder ${ }^{\mathrm{d}}$, Vanessa Stauch ${ }^{\mathrm{e}}$, Beat Lehmann ${ }^{\mathrm{f}}$, Manfred Morari ${ }^{\mathrm{a}}$ \\ a Automatic Control Laboratory, ETH Zurich, Physikstrasse 3, 8092 Zurich, Switzerland \\ ${ }^{\mathrm{b}}$ Department of Engineering, Universitá degli Studi del Sannio, Benevento, Italy \\ ${ }^{c}$ Institute of Mechanical Engineering, EPFL Lausanne, Switzerland \\ d Siemens Building Technologies, Zug, Switzerland \\ e Federal Office of Meteorology and Climatology (MeteoSwiss), Zurich, Switzerland \\ ${ }^{\mathrm{f}}$ Building Technologies Laboratory, EMPA Dübendorf, Switzerland
}

\section{A R T I C L E I N F O}

\section{Article history:}

Received 23 June 2011

Received in revised form 9 September 2011

Accepted 18 September 2011

\section{Keywords:}

Building climate control

Stochastic model predictive control

Energy efficiency

Chance-constrained control

\begin{abstract}
A B S T R A C T
This paper presents an investigation of how Model Predictive Control (MPC) and weather predictions can increase the energy efficiency in Integrated Room Automation (IRA) while respecting occupant comfort. IRA deals with the simultaneous control of heating, ventilation and air conditioning (HVAC) as well as blind positioning and electric lighting of a building zone such that the room temperature as well as $\mathrm{CO}_{2}$ and luminance levels stay within given comfort ranges. MPC is an advanced control technique which, when applied to buildings, employs a model of the building dynamics and solves an optimization problem to determine the optimal control inputs. In this paper it is reported on the development and analysis of a Stochastic Model Predictive Control (SMPC) strategy for building climate control that takes into account the uncertainty due to the use of weather predictions.

As first step the potential of MPC was assessed by means of a large-scale factorial simulation study that considered different types of buildings and HVAC systems at four representative European sites. Then for selected representative cases the control performance of SMPC, the impact of the accuracy of weather predictions, as well as the tunability of SMPC were investigated. The findings suggest that SMPC outperforms current control practice.
\end{abstract}

(c) 2011 Elsevier B.V. All rights reserved.

\section{Introduction}

Energy efficient management of building systems will play a major role in minimizing overall energy consumption and costs, since, worldwide, the residential and commercial sectors use 2589 Mtoe (mega tonnes of oil equivalent) in energy, which accounts for almost $40 \%$ of final energy use in the world; and in European countries, $76 \%$ of this energy goes towards comfort control in buildings - heating, ventilation and air conditioning (HVAC) [1]. Because of the long lifespan of buildings, it is urgent to increase the energy efficiency of the existing HVAC systems, i.e. to reduce energy use

\footnotetext{
* Corresponding author.

E-mail addresses: oldewurtel@control.ee.ethz.ch (F. Oldewurtel) aparisio@unisannio.it (A. Parisio), colin.jones@epfl.ch (C.N. Jones), gyalistras@control.ee.ethz.ch (D.Gyalistras), markus.gwerder@siemens.com (M. Gwerder), vanessa.stauch@meteoswiss.ch (V. Stauch), beat.lehmann@empa.ch (B. Lehmann), morari@control.ee.ethz.ch (M. Morari).
}

and utility costs while guaranteeing comfort for the building's occupants.

This work focuses on an individual building zone or room and considers Integrated Room Automation (IRA) [2], which uses both high energy cost actuators (e.g. chillers, gas boilers, conventional radiators) and low energy cost actuators (e.g. blind operation and evaporative cooling) for heating and cooling. The aim is to extend the use of low energy cost actuators by making use of the thermal storage capacity of the building.

The building dynamics are slow and the building is subject to intermittent disturbances, i.e. the weather as well as the building's appliances and occupants, who generate heat, $\mathrm{CO}_{2}$, and set demands for temperature, illuminance and air quality. This gives rise to a constrained control problem (because of the occupants' comfort requirements as well as the limited capacity of the actuators) and the goal is to use weather predictions in order to be able to make use of the thermal storage capacity of a building appropriately. Model Predictive Control (MPC) is an ideal framework to tackle this problem. 
The basic idea of MPC is to exploit a model of the process to predict the future evolution of the system and to compute control actions by optimizing a cost function depending on these predictions; the aim being to extend the use of low energy cost actuators for controlling the building and meeting the occupants' requirements whereas to avoid the use of high energy cost actuators as much as possible.

However, the performance of such an approach depends on the accuracy of the weather predictions which have an inherent uncertainty. Therefore, a tractable stochastic MPC approach for building climate control was developed, that explicitly takes into account the uncertainty in the problem in order to improve the control performance.

The presented work was undertaken as part of the project OptiControl [3], which aims at developing predictive control strategies that use weather and occupancy forecasts to increase the energy efficiency in building climate control while maintaining high user comfort and limiting peak electricity demand.

\subsection{Literature review}

The use of disturbance predictions, in particular weather predictions, for building climate control has been investigated in several works [4-8,2]. A link to the cited papers and a more extensive bibliography can be found on the OptiControl website [9]. In these studies the predictive strategies are shown to be more efficient when compared to conventional, non-predictive strategies for thermal control of buildings. In [7] the authors compared different predictive controllers taking into account weather predictions with a non-predictive strategy for a solar domestic hot water system. The simulation results showed that in particular for a small storage tank, the predictive control strategies achieved a lower energy cost compared to the non-predictive strategy. In $[10,11,8]$ the use of a short-term weather predictor based on observed weather data for the control of active and passive building thermal storage was explored. The predicted variables included ambient air temperature, relative humidity, and solar radiation. In [12] the implementation of MPC for a chilled water plant was investigated. A predictive control strategy using a forecasting model of outdoor air temperature was investigated in [6] for intermittently heated radiant floor heating systems. The experimental results showed that the predictive control strategy saved between $10 \%$ and $12 \%$ energy during the cold winter months compared to the existing conventional control strategy. In [13] the authors described the testing of MPC for a heating system on a real building in Prague. Energy savings in a predictive Integrated Room Automation (IRA) were investigated in [2]. The proposed model predictive strategy manipulated the passive thermal storage of the building based on predicted future disturbances while respecting comfort bounds for the room temperature. The predictive control outperformed the non-predictive control because the room temperature could be kept within its comfort bounds with minimum energy, i.e. low energy cost actuators were exploited as much as possible. The effect of automated blinds and lighting control on heating and cooling requirements were studied in [5]; the authors investigated the reduction of the annual primary energy usage in building climate control for the case of Rome. In the study of [4], the influence of occupant behavior on energy consumption was investigated in a single room occupied by one person. The simulated occupant could manipulate six controls, such as turning on or off the heat and adjusting clothing. The simulation results showed that occupant behavior significantly affects the energy consumption in the room.

In conclusion, from the literature it can be learned that predictive control strategies as well as including automated blinds and lighting in the control action provide potential benefits for energy efficient building climate control.

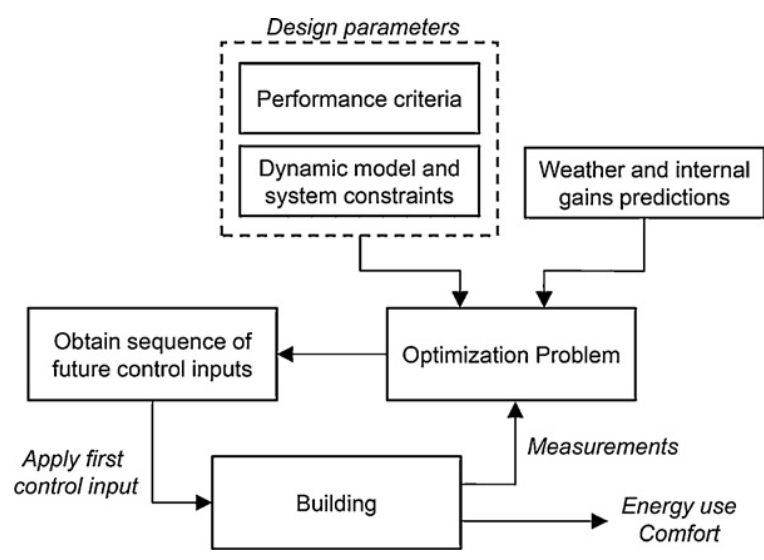

Fig. 1. MPC scheme for building climate control.

The reasons for MPC in buildings being rarely used until now are primarily the difficulties/costs of obtaining a model of an (individual) building that can be used in the MPC controller and the fact that energy costs played a minor role in the past. Using the energy savings potential of buildings by applying MPC has however become more realistic recently due to several developments: there is a drastic increase in computational power as well as the possibility to shift computations to external servers/clouds. The use of simulation tools in building planning are becoming standard and can help to obtain models for the MPC controller. The quality of weather predictions is increasing and hence its usefulness for building climate control. Energy costs are rising, and finally, there is a desire to handle time-varying electricity prices and the possibility of MPC to do so.

\subsection{Main idea and outline of the paper}

The paper aims at introducing the possibilities and new developments in Stochastic MPC to the building research and development community; it is organized as follows. Section 2 explains the basic concepts of MPC with emphasis on the use for building climate control. Section 3 describes the control strategies as well as a benchmark for building climate control that are used in the presented study. Also the newly developed Stochastic MPC approach is introduced here. In Section 4 the MPC implementation is explained in detail and the differences between the MPC options are described. Section 5 reports on the performance of the proposed controllers and, in Section 6, simulation results are presented and discussed.

\section{Introduction to model predictive control for building climate control}

MPC is a simple and satisfyingly intuitive approach to constrained control that has been successfully applied in many areas over the last decades [14,15]. During each sampling interval, a finite horizon optimal control problem is formulated and solved over a finite prediction horizon. The result is a trajectory of inputs and states into the future satisfying the dynamics and constraints of the building while optimizing some given criteria.

In terms of building climate control, this means that at the current point in time, a heating/cooling, etc. plan is formulated for the next several hours to days, based on predictions of the upcoming weather conditions, see Fig. 1. Predictions of any other disturbances (e.g., internal gains), as well as time-dependencies of the control costs (e.g., dynamic electricity prices) or of the constraints (e.g., thermal comfort range) can be readily included in the optimization. The first step of the control plan is applied to the building, determining the setting of all the heating, cooling and ventilation 
Table 1

Common types of cost functions.

\begin{tabular}{ll}
\hline Cost function type & Mathematical description \\
\hline Quadratic cost & $l_{k}\left(x_{k}, u_{k}\right)=x_{k}^{T} Q x_{k}+u_{k}^{T} R u_{k}$ \\
Linear cost & $l_{k}\left(x_{k}, u_{k}\right)=c^{T} u_{k}$ \\
Probabilistic cost & $l_{k}\left(x_{k}, u_{k}\right)=\mathbb{E}\left[g_{k}\left(x_{k}, u_{k}\right)\right]$ \\
\hline
\end{tabular}

actuators, then the procedure is repeated at the next time instant. By this receding horizon approach feedback is introduced into the system, since the new optimal control problem solved at the beginning of the next time interval is a function of the new measured state at that point in time and hence of any disturbances that have meanwhile acted on the building.

A generic MPC framework is given by the following finitehorizon optimization problem:

$\mathrm{J}\left(x_{0}\right)=\min _{u_{0}, \ldots, u_{N-1}} \sum_{k=0}^{N-1} l_{k}\left(x_{k}, u_{k}\right) \quad$ Cost function

subject to

$\left(x_{k}, u_{k}\right) \in \mathcal{X}_{k} \times \mathcal{U}_{k}$

Constraints

$x_{0}=x$

Current state

$x_{k+1}=f\left(x_{k}, u_{k}\right)$

Dynamics

where $N$ is the prediction horizon, $\mathcal{X}_{k}$ and $\mathcal{U}_{k}$ are the constraint sets respectively for states $x_{k}$ and inputs $u_{k}$ at time step $k$. The cost function and the constraints are the main pieces of the MPC design, the current state is used as the initial state for control predictions, and the dynamics of the system have to be modeled to a reasonable precision such that a good control performance is achieved. In the following, a brief explanation of each of the four components in the above MPC formulation is provided.

\subsection{Cost function}

The cost function describes the desired behavior. This generally serves two purposes:

- Stability. It is common to choose the structure of the cost function such that the optimal cost forms a Lyapunov function for the closed-loop system, and hence will guarantee stability. ${ }^{1}$ In practice, this requirement is generally relaxed for stable systems with slow dynamics such as buildings, which leaves the designer free to select the cost strictly on a performance basis.

- Performance target. The cost describes generally, but not always, a combination of performance targets; and different cost weights for the different targets can be used to specify a preference for one closed-loop behavior over another, in building climate control, e.g., minimizing energy or maximizing comfort.

Several common cost functions are in use, the majority of which are convex, which results in a simple optimization problem to solve. Some common choices, which are also listed in Table 1, are:

- Quadratic cost. The relative weighting between the states and the inputs, i.e. the choice of the matrices $Q$ and $R$ provides a trade-off between regulation quality and input energy. If the system has no constraints, or the constraints are not active, then such a cost will

\footnotetext{
1 The given formulation would have to be changed slightly for guaranteeing stability.
}

Table 2

Common types of constraints.

\begin{tabular}{ll}
\hline Constraints type & Mathematical description \\
\hline Linear constraint & $A x_{k} \leq b$ \\
Convex quadratic constraint & $\left(x_{k}-\bar{x}\right)^{T} Q\left(x_{k}-\bar{x}\right) \leq 1, Q \succcurlyeq 0$ \\
Chance constraint & $\mathbb{P}\left[A x_{k} \leq b\right] \geq 1-\alpha, \alpha \in(0,0.5]$ \\
Second order cone constraint & $\left\|A x_{k}+b\right\|_{2} \leq C x_{k}+d$ \\
Switched constraint & if condition, then $A_{1} x_{k} \leq b_{1}$ else $A_{2} x_{k} \leq b_{2}$ \\
Nonlinear constraint & $h\left(x_{k}, u_{k}\right) \leq 0$ \\
\hline
\end{tabular}

be equivalent to the cost of the Linear Quadratic Regulator/Linear Quadratic Gaussian controller (classic optimal control problem).

- Linear cost. If one wishes to minimize 'amounts', outliers, or economically motivated signals, then the linear cost function is more suitable than the quadratic one. This cost function would also be a common choice for minimizing energy consumption of buildings.

- Probabilistic cost. If the system is subject to random disturbances, then one may choose to minimize the expected value of a function $g_{k}\left(x_{k}, u_{k}\right)$, where $g_{k}\left(x_{k}, u_{k}\right)$ can for example be a quadratic or linear cost function as introduced above.

\subsection{Constraints}

The ability to specify constraints in the MPC formulation and have the optimization routine handle them directly is the key strength of the MPC approach. Many different types of constraints are used in practice, an overview of common types suitable for building climate control is given in Table 2 .

- Linear constraint. This is the most common type of constraint and is used to put upper and/or lower bounds on variables. Linear constraints are the easiest to handle when solving optimization problems and can also be used to approximate any convex constraint to an arbitrary degree of accuracy.

- Convex quadratic constraint. This type of constraint is used to bound a variable to be within an ellipsoid (i.e. a higherdimensional form of an ellipse). In building climate control, this type of constraint would arise, e.g., when bounding the sum of input energy amongst several actuators.

- Chance constraint. If uncertainty is involved in the problem, this type of constraint is used to formulate that its condition has to be fulfilled with a predefined probability. Since an optimization problem can only be solved if all variables are deterministic, chance constraints need to be reformulated into deterministic constraints.

- Second order cone constraint. This type of constraint is called conic, since the feasible region of the constraint has the form of a cone. Second order cone constraints can - under special circumstances - result from reformulations of chance constraints.

- Switched constraint. This type of constraint comprises a set of constraints, where each one is relevant only if a predefined condition is met. This is a common type of constraint in hybrid systems, i.e. systems that exhibit both continuous and discrete time behavior.

- Non-linear constraint. This type of constraint comprises any type of constraint that does not fit into the above categories, where $h\left(x_{k}, u_{k}\right)$ can be any nonlinear function. In general, it is very difficult to handle this type of constraint when solving optimization problems.

\subsection{Current state}

The system model is initialized to the measured current state of the building and all predictions begin from the system in this initial state. If some states cannot be measured but are observable, a Kalman filter would commonly be used for state estimation. 


\subsection{Dynamics}

The system model is a critical piece of the MPC controller. In the presented investigation a bilinear model was used, which will be derived in Section 4.2.

\section{Control strategies and benchmarks for building climate control}

In this section, three different control strategies are presented that are compared in the investigation: Rule-Based Control (RBC), Deterministic MPC (DMPC), and Stochastic MPC (SMPC). RBC is the current control practice and is therefore used as a benchmark. The control strategies under investigation are DMPC, which is a standard MPC formulation and SMPC, which is a newly developed MPC strategy for the purpose of building climate control that can handle the uncertainties resulting from the use of weather forecasts. All control strategies are described below. How the costs and constraints are defined for the MPC strategies is detailed in Section 4. A second benchmark is given by the so-called Performance Bound $(\mathrm{PB})$, which is defined as optimal control with perfect information, in particular with a perfect weather prediction, i.e. the realization is equal to the prediction. This is not an (implementable) controller but a concept, which is used as a benchmark.

\subsection{Control strategies}

\subsubsection{Rule-Based Control (RBC)}

The current control practice in Integrated Room Automation is RBC. RBC determines all control inputs based on a series of rules of the form "if condition, then action". The conditions and actions are usually associated with numerical parameters (e.g., threshold values) that need to be chosen. A good performance of RBC critically depends on a good choice of rules and associated parameters.

\subsubsection{Deterministic MPC (DMPC)}

DMPC is the standard MPC approach that is used in virtually all commercial MPC applications. It uses the imperfect/uncertain weather forecast and takes its control decision under the assumption that the predictions are correct (i.e. equal to certain). Therefore, it is also often called Certainty Equivalence Control. In the investigation DMPC was computed with a prediction horizon of $24 \mathrm{~h}$ and an hourly time step.

\subsubsection{Stochastic MPC (SMPC)}

The SMPC formulation proposed here is very well suited for the purpose of building climate control. This is due to the strategy's two key elements: first, the strategy directly accounts for the uncertainty in the weather forecast and second, it allows to formulate so-called chance-constraints, i.e. to enforce constraints to be fulfilled with a predefined probability, similarly as it is required in the building standards for room temperature constraints [16].

The presented approach can in principle also be extended to taking into account uncertainty in occupancy predictions if the necessary occupancy data are available and a model for the prediction error is found, which leads to a Gaussian uncertainty (similarly as for the weather predictions, see Section 4.1.2). In the present work, occupancy is however assumed to be perfectly predicted and the focus is on the consideration of uncertainty in weather predictions.

In the next two sections the two components of SMPC are detailed: the affine disturbance feedback, that accounts for the uncertainty, and the reformulation of the chance constraints for achieving a tractable optimization problem.
3.1.3.1. Affine disturbance feedback. Consider the following discrete-time linear model

$x_{k+1}=A x_{k}+B u_{k}+E w_{k}$,

where $x_{k}$ is the state at time $k, u_{k}$ is the input, and $w_{k}$ is the unknown disturbance acting on the system. We choose the input applied at time $k$ in the future to be an affine function of the as yet unknown disturbances, that will act on the system between now (time 0 ) and time $k$ :

$u_{k}:=\sum_{j=0}^{k-1} M_{k, j} w_{j}+h_{k}$,

where the matrices $M_{k j}$ and the vector $h_{k}$ are decision variables. This means that the predicted inputs are responsive to the as yet unknown disturbances.

It should be emphasized that since the MPC controller is applied in a receding-horizon fashion only the first step of the predicted inputs is applied to the system and the first predicted input does not depend on any disturbance. Only the predicted inputs of the steps $2 \ldots N-1$ are responsive to the disturbances; these inputs are however never applied to the system, but are only used as a computational procedure in order to make good decisions at the current time step.

The main benefit of using this affine disturbance feedback formulation is that the resulting optimization problem can be formulated as a convex problem, so that it can be solved using common commercial codes while providing a very good performance $[17,18]$.

The main limitation of this formulation is the added computational complexity of optimizing over $M_{k, j}$ and $h_{k}$ rather than just the inputs $u_{k}$. This can however be mitigated by, e.g., restricting the degrees of freedom of the matrices $M_{k, j}$ or by optimizing over the average of a small number of pre-computed matrices. These techniques have been shown to be effective in building climate control while significantly reducing the computational effort $[21,20]$.

3.1.3.2. Chance constraints. The disturbances acting on the buildings are caused by weather, internal gains (occupancy and equipment), and occupant behavior. Occupant behavior can have a big impact on the performance of a control strategy. However, particularly in the case of IRA, users are not likely to act directly on the subsystems (e.g., manipulate radiator valves), but instead communicate their wishes (such as changes in comfort parameters) to the control system via an interface. The controller should then carry out the corresponding actions and adjust its strategy accordingly. Hence, disturbances due to occupant behavior were not further considered. For internal gains it was assumed throughout this investigation, that a corresponding schedule is perfectly known in advance and, therefore, it is also not further addressed in this paper. Instead, the focus of this paper is on how to incorporate information about the upcoming weather. The use of weather forecasts, which are subject to errors, is considered; i.e. due to the use of weather forecasts there is some inherent uncertainty in the problem.

In order to cope with this uncertainty, a chance-constrained formulation is employed, in which the future state of the building is required to satisfy the constraints only with a given probability:

$\mathbb{P}\left[A x_{k} \leq b\right] \geq 1-\alpha$.

This formulation has significant benefits for building climate control:

- the European standards specify that comfort bounds on room temperature do not need to be guaranteed at all times, but may be violated for a small fraction of time during the year, e.g., in 
extreme weather situations [16]. By using chance constraints, a similar behavior can be encoded directly in the controller;

- when combined with the affine disturbance feedback discussed in the previous section, it is possible to formulate the resulting optimization as a second order cone problem. Such problems are convex and can be readily solved by existing commercial codes, although they can be fairly computationally expensive at larger scales.

The remainder of this document will refer to the Stochastic MPC approach in which comfort bounds are expressed as chance constraints and future control input signals are parameterized in terms of an affine disturbance feedback as SMPC. A more detailed description of the SMPC approach can be found in $[18,21]$.

\subsection{Theoretical benchmark}

\subsubsection{Performance Bound (PB)}

PB is defined as optimal control with perfect knowledge of both the system dynamics as well as all future disturbances acting upon the system. For analysis purposes, it is possible to formulate such a problem for a given building and a given year after the weather has been recorded and hence is known. PB is itself not a controller but rather a concept, that can serve as a benchmark for the investigated control strategies. In order to compute PB, the same MPC algorithm was used in the investigation as for DMPC, but with perfect weather predictions. This means that $\mathrm{PB}$ is a theoretical construct to determine the performance limit of DMPC (in the ideal case of perfect weather prediction). To compute $\mathrm{PB}$, a prediction horizon of seven days and a control horizon (i.e. the number of time steps that control inputs are applied in open-loop) of three days were used.

\section{Implementation of MPC}

In this section, it is explained how the various inputs to the controller (weather predictions, local weather and building measurements, building model data, etc.) are translated to a mathematical structure, that can be processed by standard optimization software. An overview is given in Fig. 2. This picture also gives an outline of this section.

\subsection{Step 1: weather prediction at building site}

\subsubsection{Weather forecast}

The weather predictions were given by archived forecasts of the (deterministic) numerical weather prediction model COSMO7 operated by MeteoSwiss [22]. The forecast data comprised the outside air temperature, the wetbulb temperature and the incoming solar radiation. At the time of the analysis, COSMO-7 delivered hourly predictions for the next three days with an update cycle of $12 \mathrm{~h}$ and a horizontal grid mesh size of $6.6 \mathrm{~km} \mathrm{[23].} \mathrm{For} \mathrm{this} \mathrm{study,}$ four meteorological measurement sites in different European countries were chosen in order to represent different climatic zones in Europe. The list can be found in Table 3.

\subsubsection{Error model for weather forecast}

The major challenge from a control point of view of using numerical weather predictions lies in their inherent uncertainty due to the stochastic nature of atmospheric processes, the imperfect knowledge of the weather model's initial conditions, as well as modeling errors. The actual disturbance acting on the building can be decomposed as

$v_{k}=\bar{v}_{k}+\tilde{v}_{k}$,

where $\bar{v}_{k}$ is the COSMO-7 weather forecast and $\tilde{v}_{k}$ is the prediction error at each time step $k$. In order to improve the estimation

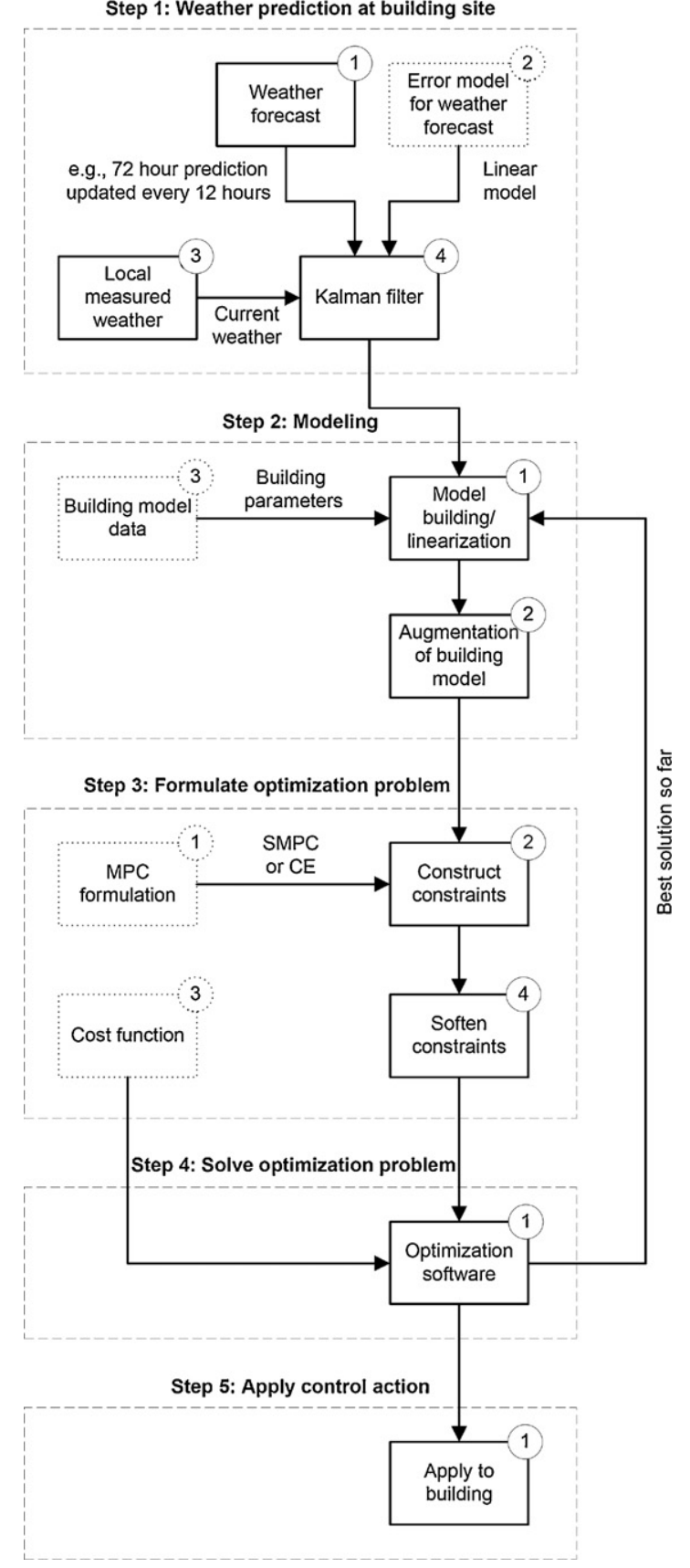

Fig. 2. Decision flow of the Model Predictive Control strategy. The parts that have to be designed a priori are in dotted boxes.

of future disturbances acting on the building, the following autoregressive model driven by Gaussian noise was identified based on the archived weather forecasts and corresponding in situ measurements

$\tilde{v}_{k+1}=F \tilde{v}_{k}+K w_{k}$.

It was assumed that $w_{k}$ follows a Gaussian distribution, $w_{k} \sim \mathcal{N}(0, I)$. Testing the randomness of residuals showed that the goodness of fit was satisfactory for all investigated cases, i.e. autocorrelation coefficients for the residuals did not differ significantly from zero.

\subsubsection{Local measured weather}

For the local measured weather, archived measurements of the Swiss Meteorological Network (SwissMetNet) at the same sites as given in Table 3 were used. Having the sites for the weather 
Table 3

Sites for the weather measurements with a short description of the ambient weather and climate and their geographical heights.

\begin{tabular}{|c|c|c|c|c|}
\hline Country & Site name & Abbrev. & Weather and climate & Height (masl) \\
\hline \multirow[t]{2}{*}{ Switzerland } & $\begin{array}{l}\text { Zürich- } \\
\text { Fluntern }\end{array}$ & SMA & $\begin{array}{l}\text { Swiss plateau climate with } \\
\text { typical inversion conditions }\end{array}$ & 556 \\
\hline & Lugano & LUG & $\begin{array}{l}\text { Representative for the Swiss } \\
\text { climate south of the Alps }\end{array}$ & 273 \\
\hline France & $\begin{array}{l}\text { Marseille } \\
\text { Marignane }\end{array}$ & MSM & Mediterranean climate & 5 \\
\hline Austria & $\begin{array}{l}\text { Wien } \\
\text { Hohe Warte }\end{array}$ & WHW & Plane with continental climate & 209 \\
\hline
\end{tabular}

forecasts and the local measured weather coincide means that there is no spatial error between the building and the weather station.

\subsubsection{Kalman filter}

In the presented setup, a Kalman filter is used to update the predictions arriving every $12 \mathrm{~h}$ on an hourly basis with the incoming new measurements, e.g. [24]. In a realistic setting the use of a Kalman filter would also be beneficial in order to eliminate the systematic error due to the fact that the building is not situated directly at the weather station site as well as due to the environment of the building itself (shadow from other buildings, trees, etc.). Furthermore, a Kalman filter would be used if not all system states can be measured. In this study, it is however assumed that all states are measured.

\subsection{Step 2: modeling}

\subsubsection{Building modeling}

There is a large amount of computer-aided modeling tools (e.g., TRNSYS [25], EnergyPlus [26]), however, these are designed mainly for estimating the energy usage of a building, and cannot be readily used for control. Instead, for this study, a thermal ResistanceCapacitance (RC)-network was constructed and employed.

For computing the building-wide energy use it is common practice to sum the energy uses of single rooms or building zones [2]. Following this approach, the focus was on the dynamics of a single room. First, the building thermal dynamics are explained in detail and then, the different actuators are introduced.

Illuminance and $\mathrm{CO}_{2}$ concentration were modeled by instantaneous responses since the time constants involved were much smaller than the hourly time step employed for the modeling and simulations; modeling details of these are omitted. The interested reader can find the details on this in [27].

The principle of the thermal dynamics modeling can easily be described with a small example as given in Fig. 3. The room can be thought of as network of first-order systems, where the nodes are the states $x$ and these are representing the room temperature or the
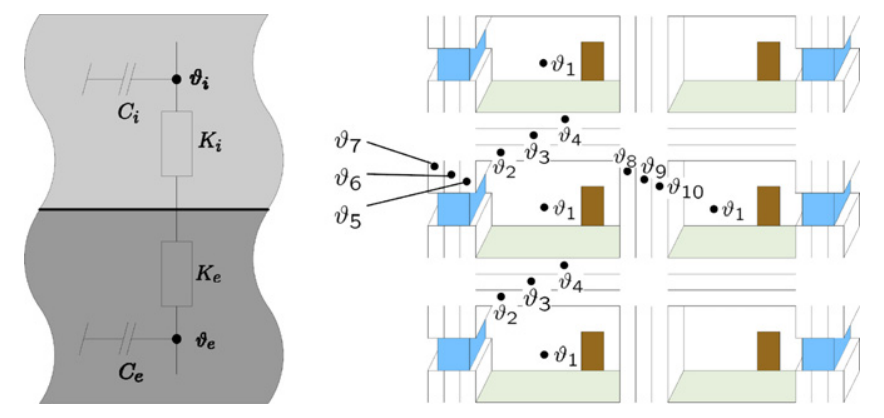

Fig. 3. Heat transmission between nodes. The modeling is based on the description of heat transmission between nodes (left) that are representing the temperatures at different locations in the building (right). temperatures in the walls, floor or ceiling. Then the heat transfer rate is given by

$$
\underbrace{\frac{d Q}{d \vartheta_{i}}}_{C_{i}} \cdot \frac{d \vartheta_{i}}{d t}=\underbrace{A \cdot U_{i e}}_{K_{i e}}\left(\vartheta_{e}-\vartheta_{i}\right)
$$

where $t$ denotes the time, $\vartheta_{i}$ and $\vartheta_{e}$ are the temperatures in layers $i$ and $e$ respectively, $Q$ is thermal energy, $C_{i}$ denotes the thermal capacitance of layer $i, A$ is the cross-sectional area, and $U_{i e}$ the heat transmission coefficient. The total heat transmission coefficient $K_{i e}$ is computed as

$$
\frac{1}{K_{i e}}=\frac{1}{K_{i}}+\frac{1}{K_{e}}
$$

where the heat transmission coefficients $K_{i}$ and $K_{e}$ depend on the materials of $i$ and $e$ as well as on the cross sectional area of the heat transmission.

For each node, i.e. state, such a differential equation as in (10) is formulated. Control actions were introduced as direct heat flux into nodes or by assuming that select resistances were variable. For example, solar heat gains and luminous flux through the windows were assumed to vary linearly with blinds position, i.e. the corresponding heat transfer coefficients were multiplied with an input $u \in[0,1]$. This leads to a bilinear model, i.e. bilinear in state and input as well as in disturbance and input. A detailed description of the building model can be found in [27].

Concerning the actuators, five variants of HVAC systems typical for IRA were investigated, which are listed in Table 4 . The delivered heating or cooling power, the used air change rates, as well as lighting and blind positioning correspond to the control inputs $u$. Typically, some of the actuator efficiencies depend on the weather (e.g., the efficiency of the cooling tower depends on the wetbulb temperature) and the actuators have different response characteristics (e.g., the response of the room temperature to changes in the TABS is very slow whereas the response to heating with mechanical ventilation is very fast). Finally, the actuators have different energy costs. Further details on the simulation setup as well as the complete RC model including its numerical values can be found in [28]. Based on this, the room dynamics can be described as

$\vartheta_{k+1}=A_{(\vartheta)} \vartheta_{k}+B_{(u)} u_{k}+B_{(v)} v_{k}+\sum_{i=1}^{m}\left[\left(B_{(v u, i)} v_{k}+B_{(x u, i)} \vartheta_{k}\right) u_{k, i}\right]$

where $\vartheta_{k} \in \mathbb{R}^{n}$ is the state, $u_{k} \in \mathbb{R}^{m}$ is the input, and $v_{k} \in \mathbb{R}^{p}$ is the weather input (outside temperature, wetbulb temperature, solar radiation) at time step $k$, and the matrices $A_{(\vartheta)}, B_{(u)}, B_{(v)}, B_{(v u, i)}$, and $B_{(x u, i)}$ are of appropriate sizes. The sampling time is $1 \mathrm{~h}$. The bilinear part is due to the blinds (i.e. by changing the corresponding actuator, the heat transfer between the room node and the outside temperature is changed) and due to the cooling tower (i.e. by changing the corresponding actuator, the cooling with the cooling tower, which is depending on the difference between room temperature 
Table 4

Overview of five variants of HVAC systems.

\begin{tabular}{|c|c|c|c|c|c|}
\hline Automated subsystems & 1 & 2 & 3 & 4 & 5 \\
\hline Blinds & $\sqrt{ }$ & $\sqrt{ }$ & $\sqrt{ }$ & $\sqrt{ }$ & $\sqrt{ }$ \\
\hline Electric lighting & $\sqrt{ }$ & $\sqrt{ }$ & $\sqrt{ }$ & $\sqrt{ }$ & $\sqrt{ }$ \\
\hline Mech. ventilation flow, heating, cooling & - & $\sqrt{ }$ & $\sqrt{ }$ & $\sqrt{ }$ & $\sqrt{ }$ \\
\hline Natural ventilation heating/cooling (night-time only) & - & - & - & $\sqrt{ }$ & - \\
\hline Cooled ceiling (capillary tube system) & $\sqrt{ }$ & $\sqrt{ }$ & - & - & - \\
\hline Free cooling with wet cooling tower & $\sqrt{ }$ & $\sqrt{ }$ & - & - & $\sqrt{ }$ \\
\hline Radiator heating & $\sqrt{ }$ & $\sqrt{ }$ & - & - & - \\
\hline Floor heating & - & - & - & $\sqrt{ }$ & - \\
\hline Thermally activated building systems for heating/cooling & - & - & - & - & $\sqrt{ }$ \\
\hline
\end{tabular}

and wetbulb temperature, is changed). The overall building model was validated by building experts [27] and its dynamic response compared to simulations with TRNSYS, which is a well known simulation software for buildings and HVAC systems. The results showed good consistency of the calculated room temperatures [27].

Since the dynamic behavior of the building is bilinear between inputs, states and weather parameters, the dynamic equations of the MPC problem result in a non-convex optimization, which can be difficult to solve. The approach that is taken is a form of Sequential Linear Programming (SLP) for solving non-linear problems in which the system is iteratively linearized around the current solution, the optimization problem is solved and it is repeated until a convergence condition is met [29].

\subsubsection{Augmentation of building model}

In order to be able to better account for the uncertainty in the weather prediction, the building model in (12) is augmented by the error model in (9). Assuming that the building model was linearized and that the matrix $B_{(u \mid k)}$ contains all input matrices yields

$$
\begin{aligned}
& \underbrace{\left[\begin{array}{l}
\vartheta_{k+1} \\
\tilde{v}_{k+1}
\end{array}\right]}_{x_{k+1}}=\underbrace{\left[\begin{array}{ll}
A_{(\vartheta)} & B_{(v)} F \\
\mathbf{0} & F
\end{array}\right]}_{A}[\underbrace{\left[\begin{array}{l}
\vartheta_{k} \\
\tilde{v}_{k}
\end{array}\right]}_{x_{k}}+\underbrace{\left[\begin{array}{l}
B_{(u \mid k)} \\
\mathbf{0}
\end{array}\right]}_{B_{k}} u_{k}+\underbrace{\left[\begin{array}{l}
B_{(v)} \\
\mathbf{0}
\end{array}\right]}_{H} \bar{v}_{k} \\
& +\underbrace{\left[\begin{array}{l}
B_{(v)} K \\
K
\end{array}\right]}_{E} w_{k}
\end{aligned}
$$

and hence

$\Rightarrow x_{k+1}=A x_{k}+B_{k} u_{k}+H \bar{v}_{k}+E w_{k}$.

Note that $B_{k}$ is time-varying, hence, the overall model is a timevarying linear model with the weather and internal gains prediction $\bar{v}_{k}$ as external input and uncertainty $w_{k} \sim \mathcal{N}(0, I)$.

\subsubsection{Building model data}

One problem to get such a model as in (12) is to get the parameters such that the equations as in (10) can be set up. These parameters can be either determined from the construction plan according to the materials used and their tabular values or, alternatively, the parameters can be determined via estimation methods. For this investigation, only simulation models needed to be constructed, therefore, tabular values for the materials were used. This is detailed in [27].

\subsection{Step 3: formulate optimization problem}

In this section, it is reported on how to formulate the optimization problem. For this, one needs to choose an appropriate MPC formulation, then according to this construct the constraints and formulate the cost function and finally soften the constraints.

\subsubsection{MPC formulation}

One needs to choose between the two principle MPC formulations: deterministic MPC and stochastic MPC. This choice determines the formulation of the constraints and the cost function. Hence, in the following these formulations are detailed for both choices, deterministic MPC and stochastic MPC.

\subsubsection{Construct constraints}

Two types of constraints are to be enforced: input power limits and room temperature comfort bounds (as well as bounds on illuminance and $\mathrm{CO}_{2}$ ). The formulation of the room temperature constraints is shown in detail, other constraints can be formulated in a similar fashion. Denote with $x_{t, 1}$ the first element of the state vector $x$ of (14), which is the room temperature at time $t$. Since the model (12) has been linearized, the predicted room temperature at $t$ time steps in the future is a linear function of the state now (time $t=0$ ) and the intervening inputs $u_{k}$ and weather $w_{k}$ acting on the building. As a result, the room temperature $x_{t, 1}$ at time $t$ in the future can be written as

$x_{t, 1}=L x_{0}+\sum_{k=0}^{t-1}\left(G_{k} u_{k}+V_{k} \bar{v}_{k}+W_{k} w_{k}\right)$

for appropriate vectors $L, G_{k}, V_{k}$ and $W_{k}$, which are computed directly from the output of Step 2 . The critical issue is that the disturbances $w_{k}$ are not known exactly, but they are only known to follow a standard normal distribution. Three methods for enforcing bounds on the temperature despite this disturbance were considered:

\subsubsection{Deterministic MPC.}

\section{- Deterministic MPC (DMPC)}

When using DMPC or Certainty Equivalence MPC the idea is to assume that the uncertainty $w_{k}$ takes its expected value, i.e. $w_{k}=0$. The result is a simple linear constraint of the form

$x_{t, 1}^{\min } \leq x_{t, 1}=L x_{0}+\sum_{k=0}^{t-1}\left(G_{k} u_{k}+V_{k} \bar{v}_{k}\right) \leq x_{t, 1}^{\max }$,

where $x_{t, 1}^{\min }$ and $x_{t, 1}^{\max }$ are the desired upper and lower comfort bounds, which can be time-varying, e.g., due to night-setbacks for the room temperature.

The primary limitation of DMPC is the fact that, if the weather does not actually equal the expected value, then the bound may be violated. This is most often dealt with by artificially tightening the upper and lower bounds, which provides a buffer zone and can be effective for small variances. The cost to be paid is the additional energy required to hold the room temperature further away from the bounds.

- Performance Bound (PB)

For PB the same formulation for the constraints is used as in (16). However, since there are perfect weather predictions 
available, $w_{k}$ actually takes its expected value and hence, the bounds are not violated.

\subsubsection{Stochastic MPC.}

\section{- Stochastic MPC with chance constraints}

One method of automatically determining an appropriate amount to tighten the constraints (shown for the upper bound here) is to formulate them as chance constraints as discussed in Section 3.1.3.2.

$\mathbb{P}\left[x_{t, 1}=L x_{0}+\sum_{k=0}^{t-1}\left(G_{k} u_{k}+V_{k} \bar{v}_{k}+W_{k} w_{k}\right) \leq x_{t, 1}^{\max }\right] \geq 1-\alpha$,

where $\alpha$ is the desired probability of constraint satisfaction. For the case of a standard Gaussian disturbance, a classic result by Prékopa [30] allows to re-write this probabilistic constraint as a linear condition

$x_{t, 1}=L x_{0}+\sum_{i=0}^{t-1}\left(G_{k} u_{k}+V_{k} \bar{v}_{k}\right) \leq x_{t, 1}^{\max }-\beta$,

where $\beta$ is a constant defined as

$\beta=\Phi^{-1}(1-\alpha)\left\|\left[W_{0} \ldots W_{t-1}\right]\right\|_{2}$,

where $\Phi$ is the standard Gaussian cumulative distribution function. This deterministic reformulation is exact. Despite the complex look of the above equation, it is similar to the tuning of DMPC discussed above, where the tightening is set in order to achieve a particular probability of constraint violation. While this procedure is conceptually appealing, the value $\beta$ grows quickly with the length of the prediction horizon and can hold the temperature very far away from the bounds, which costs lots of energy. This is why the procedure discussed in the next section was introduced.

- Stochastic MPC with chance constraints and affine disturbance feedback (SMPC)

A less conservative approach is a chance constraint formulation combined with affine disturbance feedback, as discussed in Section 3.1.3. In this approach, the input is set equal to an affine function of the as yet unknown weather conditions, which changes the probabilistic constraint to

$$
\begin{aligned}
\mathbb{P}\left[x_{t, 1}=\right. & L x_{0}+\sum_{k=0}^{t-1}\left(G_{k} h_{k}+G_{k} \cdot \sum_{j=0}^{k-1} M_{k, j} w_{j}+V_{k} \bar{v}_{k}\right. \\
& \left.\left.+W_{k} w_{k}\right) \leq x_{t, 1}^{\max }\right] \geq 1-\alpha,
\end{aligned}
$$

where one can see that the new optimization variables $M_{k}$ and $h_{k}$ were added. This procedure theoretically allows to mitigate the effect of the disturbance. This mitigation is, however, bounded by the amount of input energy available to the controller, since the approach requires that some input energy is allocated for compensating for the disturbance $\left(M_{k}\right)$, and some for steering the system to a desired state $\left(h_{k}\right)$.

The added complexity of this approach arises from the fact that the variable $\beta$ introduced in the previous section is now a nonlinear function of the new optimization variables $M_{k}$. The resulting optimization problem has a second order cone constraint, which is convex and therefore tractable. However, due to the large number of variables involved, solving this problem can be very time consuming and as a result somewhat impractical. For this reason, the matrices $M_{k}$ were chosen during a pre-processing step and fixed for the entire year, which turns the constraints into linear constraints.

\subsubsection{Cost function}

In this investigation the aim was to minimize the expected value of the energy use and therefore a probabilistic cost with a linear cost function was chosen

$l_{k}\left(x_{k}, u_{k}\right)=\mathbb{E}\left[c_{k}^{T} u_{k}\right]$.

The linear cost is time-dependent in order to account, e.g., for electricity prices that differ between day- and night-time.

4.3.3.1. Deterministic MPC. For DMPC, the cost function is not depending on $w_{k}$, therefore the cost function simplifies to

$l_{k}\left(x_{k}, u_{k}\right)=c_{k}^{T} u_{k}$.

4.3.3.2. Stochastic MPC. If the affine disturbance feedback is used as in the SMPC formulation, then the cost function is depending on the uncertainty $w_{j}$.

$l_{k}\left(x_{k}, u_{k}\right)=\mathbb{E}\left[c_{k}^{T}\left(h_{k}+\sum_{j=0}^{k-1} M_{k, j} w_{j}\right)\right]$.

Since the cost is linear and the distribution Gaussian, the expected value is a linear function of the mean values and therefore simplifies to

$l_{k}\left(x_{k}, u_{k}\right)=\mathbb{E}\left[c_{k}^{T} h_{k}\right]$.

Note that even though $M_{k}$ does not appear explicitly in the cost function, it appears in the constraints and influences the choice of $h_{k}$

\subsubsection{Soften constraints}

It is not always possible to satisfy all constraints of the building and so a standard relaxation-procedure, the so-called softconstraints $[14,15]$, is required, that chooses automatically which constraints are to be violated first. This is achieved by adding variables to the optimization routine which allows every constraint to be violated. These variables are however heavily penalized, which forces them to zero, i.e. to the satisfaction of all constraints, if at all possible. If this is not possible, then these additional variables give the optimizer sufficient flexibility to always find a solution that can be applied to the building. One can define the relative importance of each constraint by tuning the relevant weighting matrices and thereby have the system violate the least important first [31].

\subsection{Step 4: solve optimization problem}

Once the constraints and cost have been formulated, the resulting problem can be passed to a standard optimization routine. For this work, the commercial package CPLEX [32] was used, since it is effective for the large-scale and sparse problems that result.

\subsection{Step 5: apply control action}

The optimal solution of the formulated optimization problem consists of a sequence of planned inputs over a time horizon into the future. Only the first of these inputs is applied to the building before re-solving the entire problem at the next point in time.

\section{Performance of stochastic model predictive control in building climate control}

\subsection{Controller assessment concept}

In this section, it is reported on how the different controllers were assessed. The performance of the controllers for one simulated year is considered. Generally, the simulation of PB and RBC 


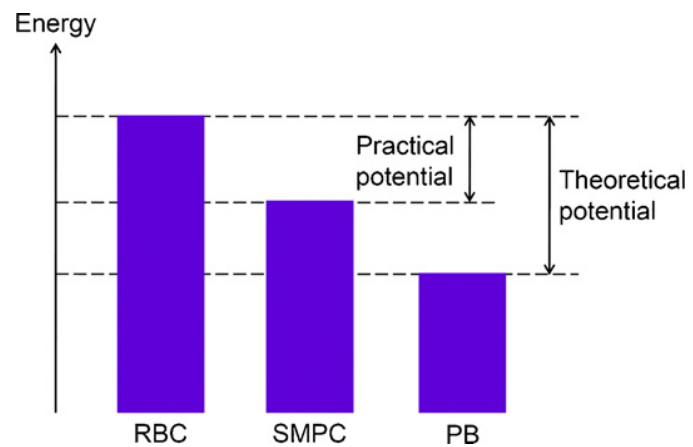

Fig. 4. Controller assessment concept. First the theoretical potential was assessed (comparison of RBC and $\mathrm{PB}$ ), then the practical potential was assessed (comparison of RBC and SMPC).

was computationally very easy and therefore it was possible to run a large number of simulations for different building setups. The simulation of DMPC and SMPC was computationally more expensive. Therefore, the investigation was done in two steps; first, a largescale simulation was carried out with PB and RBC and based on these results, DMPC and SMPC were compared to the corresponding cases with $\mathrm{PB}$ and $\mathrm{RBC}$ for some chosen example cases. The assessment concept is shown in Fig. 4 and explained below.

1 Theoretical potential: The first step consisted of the comparison of $\mathrm{RBC}$ and $\mathrm{PB}$. This was done because there is only hope for significant energy savings, if the gap between RBC and PB is large. This investigation was done in a large-scale factorial simulation study for a broad range of cases representing different buildings, HVAC systems, and weather conditions as described below. For further details see $[33,34]$. Here the aim was to answer the following questions:

Q1 Theoretical savings potential: How big are the theoretical savings potentials in IRA?

Q2 Prediction horizon length: What is a suitable prediction horizon length when applying MPC with weather predictions (depending on the building and HVAC system)?

2 Practical potential: In this investigation the performance of RBC and SMPC strategies was compared, but only for selected cases from the theoretical potential study. It was also compared with the performance of DMPC, which is expected to perform worse than SMPC since it does not take into account the uncertainty in the problem. Further details can be found in [35]. For the practical potential the aim was to answer the following questions:

Q3 Performance of DMPC: What is the performance of DMPC in IRA?

Q4 Performance of SMPC: What is the added value of SMPC in IRA?

Q5 Importance of weather predictions: What impact do weather forecasts and their quality have?

Q6 Tunability: How can MPC facilitate to choose the desired tradeoff between energy use and comfort?

In this study, the Non-Renewable Primary Energy (NRPE) usage was assessed as well as the amount and number of constraint violations of the room temperature. A reasonable violation level for room temperature as it would be tolerated according to the standards would be about $70 \mathrm{Kh} / \mathrm{a}^{2}$ [16]. In the investigation of the theoretical potential, the variants listed in Table 5 were considered. The combination of all possible variants makes in total 1280 cases. In

\footnotetext{
$21 \mathrm{Kh} / \mathrm{a}$ (=KelvinHour/Annum) corresponds to exceeding the temperature constraint by $1 \mathrm{~K}$ for $1 \mathrm{~h}$ within 1 year
}

Table 5

Overview of variants for investigation of theoretical savings potential.

\begin{tabular}{ll}
\hline Attribute & Value \\
\hline Facade orientation & North \\
& South \\
& South-east \\
& South-west \\
Construction type & Heavyweight \\
& Lightweight \\
Building standard & Swiss average \\
& Passive house \\
Window area fraction & Low \\
& High \\
Internal gains level & Low \\
& High \\
HVAC systems & 5 variants, see Table 4 \\
Locations & 4 variants, see Table 3 \\
\hline
\end{tabular}

the investigation of the practical potential the cases listed in Table 6 were considered. These cases were selected to reflect frequent and interesting building setups, with typical to large theoretical savings potentials [35]. For DMPC and SMPC a Kalman filter was applied as described in Section 4.1.4. For PB and RBC, no Kalman filter was necessary, since $\mathrm{PB}$ has a perfect prediction available and RBC does not have any prediction available. All simulations were carried out for one year and the used weather data was of the year 2007. In the following, it is detailed how the six questions $\mathbf{Q 1}$ to $\mathbf{Q 6}$ were addressed:

\section{Q1 Theoretical savings potential}

For the corresponding analysis PB and RBC were compared for 1280 cases. A case is given by one choice of the parameters listed in Table 5.

\section{Q2 Prediction horizon length}

For the corresponding analysis PB with a prediction horizon of seven days was compared with PB simulations with shorter prediction horizons for the 1280 cases.

\section{Q3 Performance of DMPC}

For the corresponding analysis DMPC was employed using weather forecasts from the COSMO-7 numerical weather prediction model. All cases from Table 6 were analyzed. PB and RBC were used as benchmarks.

\section{Q4 Performance of SMPC}

For the corresponding analysis SMPC was employed using weather forecasts from the COSMO-7 numerical weather prediction model. Six example cases from Table 6 were analyzed. PB and RBC were used as benchmarks.

\section{Q5 Importance of Weather Predictions}

This question was treated by comparing SMPC performance using COSMO-7 weather predictions, i.e. provided by a weather service, versus using $24 \mathrm{~h}$ persistence predictions, i.e. continuous recycling of the data from the last $24 \mathrm{~h}$. Persistence is a common benchmark in meteorology to assess the quality of predictions. Again, the same six example cases from Table 6 were analyzed.

Q6 Tunability

It was investigated for Building Case 1 in Table 6 how the desired comfort level can be achieved with SMPC considering the tradeoff between energy use and comfort violations. For this, the parameter $\alpha$ was varied.

\section{Results}

\subsection{Theoretical energy savings potential}

\section{Q1 Theoretical savings potential}

In the investigated 1280 cases, the reasonable amount of violations of $70 \mathrm{Kh} / \mathrm{a}$ was exceeded for many cases when using the RBC controller. It was decided to only consider amounts of violation 
Table 6

Overview of investigated cases for determining the practical savings potential.

\begin{tabular}{|c|c|c|c|c|c|c|c|c|}
\hline Building case & Location & Building standard & HVAC system & Facade orientation & Construction type & Window fraction & Internal gains & Ventil. IAQ controlled \\
\hline 1 & Lugano & sa & 2 & $\mathrm{~S}$ & High & Low & High & Yes \\
\hline 2 & Lugano & sa & 2 & $\mathrm{~S}$ & High & Low & High & No \\
\hline 3 & Marseille & sa & 2 & $\mathrm{~S}$ & High & Low & High & Yes \\
\hline 4 & Marseille & sa & 2 & $\mathrm{~S}$ & Low & Low & High & Yes \\
\hline 5 & Zurich & pa & 2 & SW & High & High & High & Yes \\
\hline 6 & Zurich & sa & 2 & SW & High & Low & High & Yes \\
\hline 7 & Zurich & pa & 2 & $\mathrm{~S}$ & High & High & High & Yes \\
\hline 8 & Zurich & pa & 2 & $\mathrm{~S}$ & Low & High & High & Yes \\
\hline 9 & Zurich & sa & 2 & $\mathrm{~S}$ & High & Low & High & Yes \\
\hline 10 & Zurich & sa & 2 & $S$ & High & Low & High & No \\
\hline 11 & Vienna & pa & 2 & SW & High & High & High & Yes \\
\hline 12 & Vienna & sa & 2 & $\mathrm{~S}$ & High & Low & High & Yes \\
\hline 13 & Zurich & sa & 3 & $\mathrm{~S}$ & High & Low & High & Yes \\
\hline 14 & Vienna & pa & 3 & SW & High & High & Low & Yes \\
\hline 15 & Lugano & sa & 4 & $\mathrm{~S}$ & High & Low & High & Yes \\
\hline 16 & Zurich & pa & 4 & $\mathrm{~S}$ & High & High & High & Yes \\
\hline 17 & Zurich & pa & 5 & $S$ & High & High & High & Yes \\
\hline 18 & Vienna & pa & 5 & $S$ & High & High & High & Yes \\
\hline
\end{tabular}

by RBC of $<300 \mathrm{Kh} / \mathrm{a}$. In this category fell 1228 of the 1280 cases. Fig. 5 shows the joint cumulative distribution of the theoretical energy savings potential (as additional NRPE use in \% of PB) and the amount of comfort violations in $\mathrm{Kh} / \mathrm{a}$. It can be seen that more than half of the considered cases show an additional NRPE use of more than $40 \%$ (rightmost brown column). Thus, for many cases there is a significant savings potential, which can potentially be exploited by SMPC. The selection of cases for the practical potential analysis was based both on common building setups and large theoretical savings potentials. The cases set is given in Table 6 .

\section{Q2 Prediction horizon length}

In Fig. 6 the average prediction horizon length (averaged over all cases from the theoretical potential analysis), which is necessary for reaching errors less than $5 \%$ compared to $\mathrm{PB}$, is plotted. The analysis is separated for different HVAC systems and the two building standards. One can see that for most systems (on average) a prediction horizon of $24 \mathrm{~h}$ is sufficient to deviate not more than $5 \%$ from the PB performance. That is why it was decided to use a $24 \mathrm{~h}$ prediction horizon for all investigated cases (except for PB simulations). The big difference in required prediction horizon

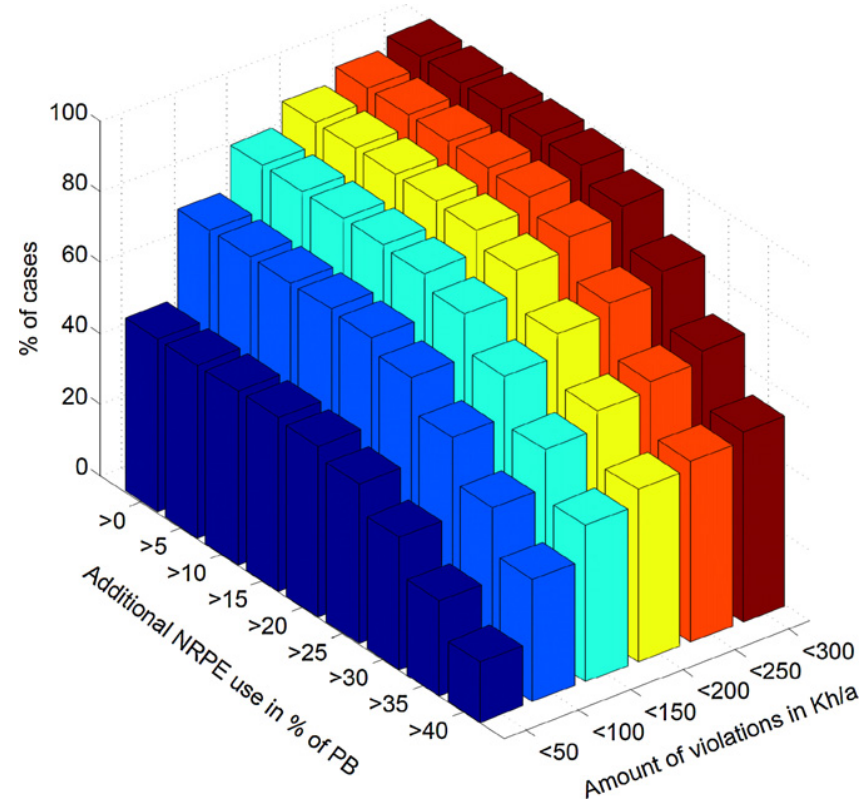

Fig. 5. Joint cumulative distribution of a particular additional energy use with RBC in \% of PB and a particular amount of violations by RBC in Kh/a. lengths that was found for HVAC system S5 can be attributed to the fact that Passive house buildings have much lower heating and cooling energy demand as compared to Swiss average buildings. Accordingly, in the latter case the TABS's thermal storage capacity becomes more important, and because TABS are thermally inert systems their predictive control requires in general much longer prediction horizons.

\subsection{Practical energy savings potential}

\section{Q3 Performance of DMPC}

The performance of DMPC in terms of NRPE use and violations for all cases in Table 6 was compared to PB and RBC and is plotted in Fig. 7. DMPC and RBC had a larger NRPE use than PB and had both many violations. When comparing RBC and DMPC, both RBC as well as DMPC exceeded $70 \mathrm{Kh} / \mathrm{a}$, but DMPC violated this threshold more clearly. The number of violations were also typically larger for DMPC. Note, for both RBC and DMPC the data points for Cases 4 and 8 are not shown because they were far beyond the axes ranges of the plots.

\section{Q4 Performance of SMPC}

In order to assess the possible added value of SMPC as compared to the simpler DMPC controller, PB, DMPC, RBC, and SMPC for the cases in Table 6 were considered. Note, for SMPC only cases $1,2,3,7,17$, and 18 were computed. The results for SMPC are also shown in Fig. 7. SMPC resulted in clearly less violations than DMPC and for the available cases also had a comparable or smaller NRPE use. The amount of violation was in all cases less than $70 \mathrm{Kh} / \mathrm{a}$.

In Fig. 8, SMPC is directly compared for the same six cases with the performance of RBC. It is shown that with SMPC the additional NRPE use for all cases can be reduced significantly compared to RBC. In four of the six cases also the amount of violations can be reduced, resulting in all cases in violations lying below the violation limit of $70 \mathrm{Kh} / \mathrm{a}$.

Figs. 9 and 10 show the resulting room temperature profiles throughout the whole year for Case 3 in Table 6 when using RBC and SMPC respectively. It can be seen that SMPC has smaller and less frequent violations than RBC. Furthermore, the diurnal temperature variations are much smaller with SMPC, which is a more favorable behavior in terms of comfort. This behavior is observable for the other cases in a similar fashion.

\section{Q5 Importance of weather predictions}

Fig. 11 depicts the performance of SMPC with persistence predictions $\left(\mathrm{SMPC}_{\text {pers }}\right.$ ) versus COSMO-7 weather predictions $\left(\mathrm{SMPC}_{\mathrm{C}}\right)$, (which was used for all investigations above). SMPC $_{\text {pers }}$ shows in all 

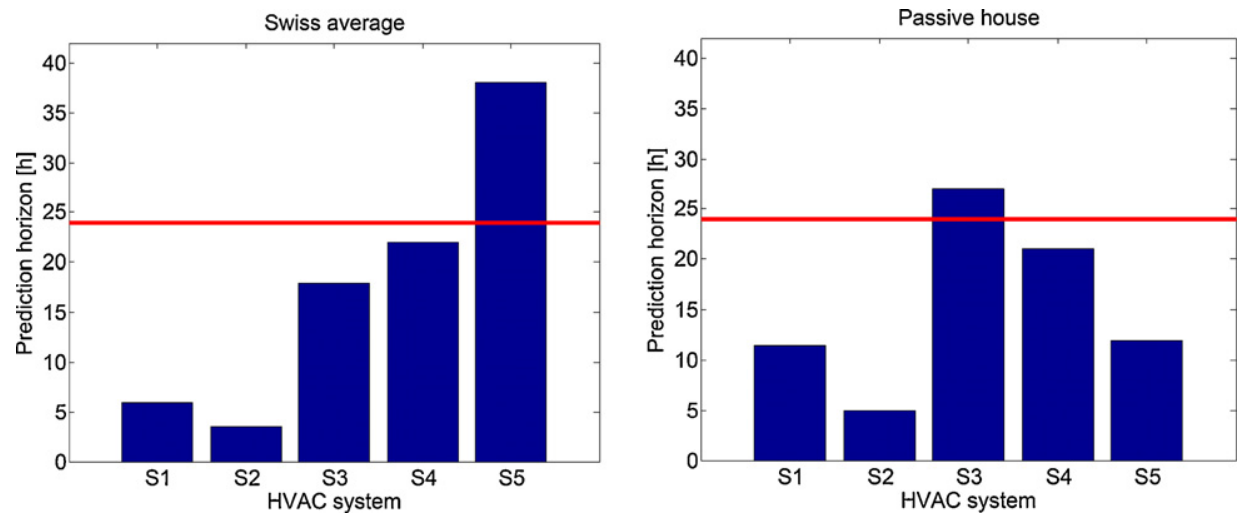

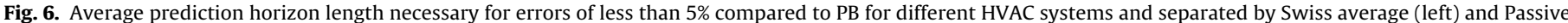
house (right).
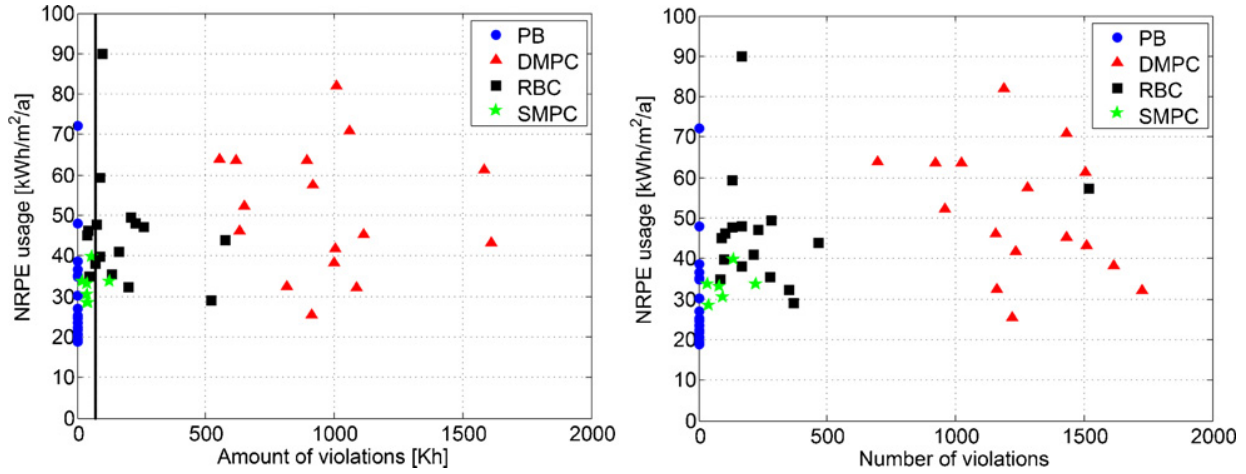

Fig. 7. Comparison of PB, DMPC, RBC and SMPC in terms of NRPE usage versus amount of violations (left) as well as number of violations (right).

cases a clearly higher NRPE use. In two cases each, $\mathrm{SMPC}_{\text {pers }}$ shows slightly less violations, equal amounts of violations, and clearly larger violations than $\mathrm{SMPC}_{C 7}$.

\section{Q6 Tunability}

For Building Case 1 from Table 6 the obtained Pareto frontier with respect to the annual NRPE use and annual amount of comfort violations is shown in Fig. 12. The curve shows a smooth behavior and it can be seen that a decrease in the amount of violations from 70 to $40 \mathrm{Kh} / \mathrm{a}$ goes along with an additional NRPE use of $10 \%$.



Fig. 8. Comparison of SMPC and RBC for Cases 1, 2, 3, 7, 17, and 18 in Table 6.

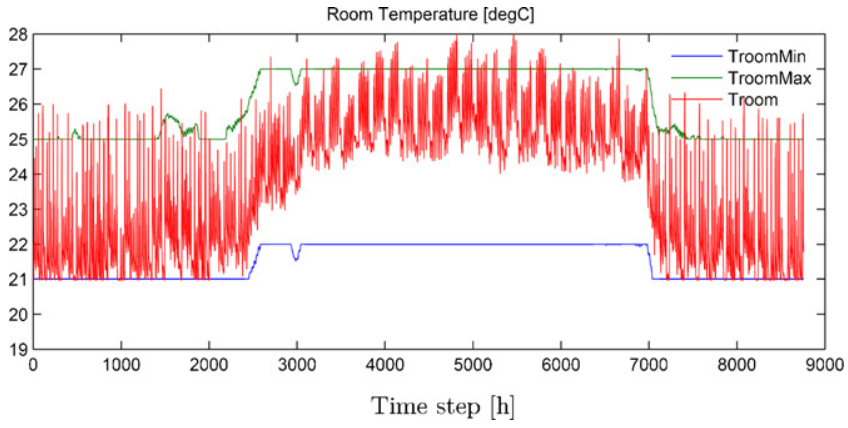

Fig. 9. Room temperature profile of Case 3 in Table 6 using RBC for year 2007.

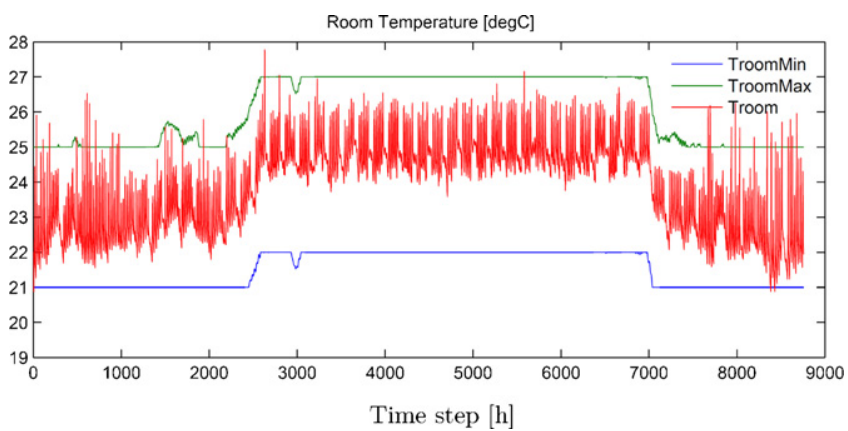

Fig. 10. Room temperature profile of Case 3 in Table 6 using SMPC for year 2007 


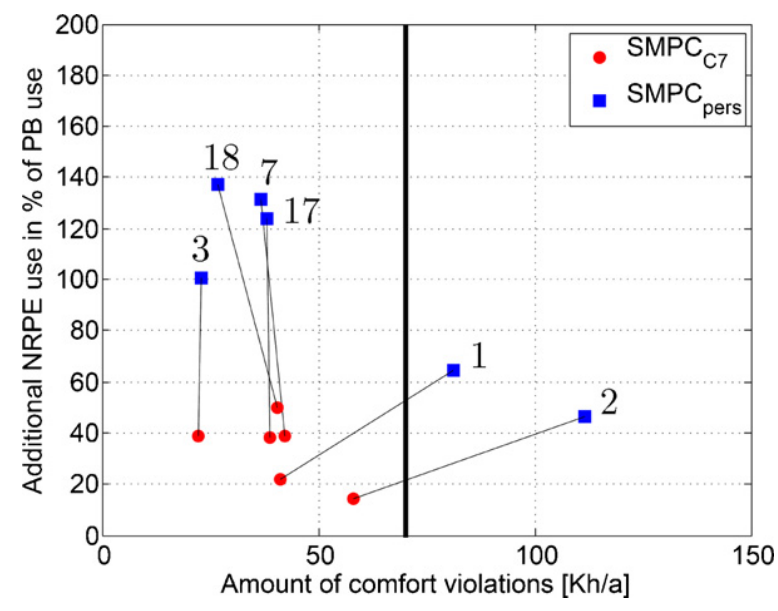

Fig. 11. Comparison of SMPC with different weather predictions for Cases 1, 2, 3, 7, 17, and 18 in Table 6.

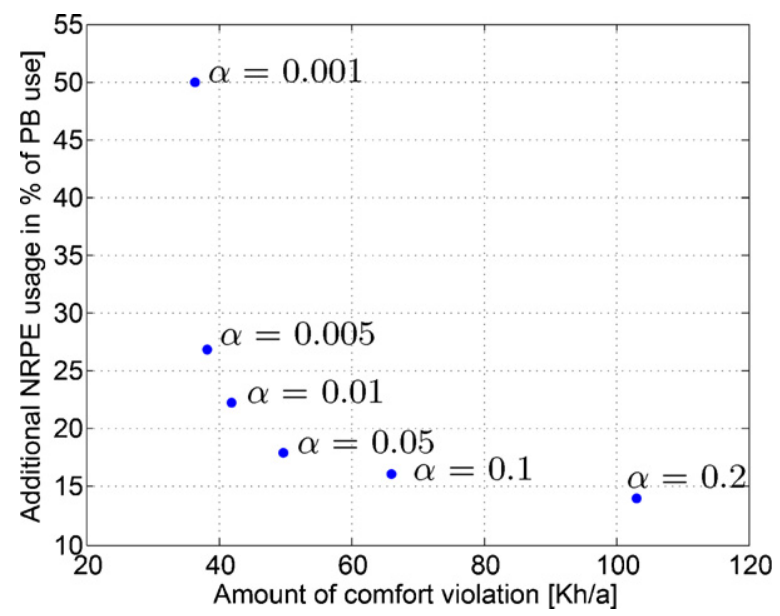

Fig. 12. Tuning of SMPC for Case 1 in Table 6. Pareto frontier of NRPE usage versus comfort violations.

\section{Conclusion}

This paper presents an investigation of the energy savings potential in Integrated Room Automation (IRA) both when comparing the current control practice (RBC) with a theoretical benchmark, the Performance Bound (PB), and when comparing it with Deterministic MPC (DMPC) and Stochastic MPC (SMPC). Unlike other works that often focus on a particular example, the energy savings potential was estimated in a large-scale simulation study for a large number of different cases varying in the building type, HVAC system, and weather conditions. The results of the large-scale simulation study, that compared the performance of PB and RBC, showed that there is a significant energy savings potential for MPC in many cases.

A newly developed SMPC strategy for building climate control was presented. This controller makes use of weather forecasts in order to compute how much energy and which low/high energy cost actuators are needed to keep the room temperature in the required comfort levels. SMPC was shown to outperform RBC, which is the current control practice, not only in terms of NonRenewable Primary Energy (NRPE) usage and thermal comfort statistics, but also in terms of advantageous room temperature dynamics. SMPC resulted in much smaller diurnal temperature variations, and this behavior can be considered more favorable since the occupants are exposed to much smaller temperature variations during the day. When comparing to DMPC, SMPC was found to be superior both in terms of NRPE usage and comfort violations. This is due to the fact that unlike DMPC, SMPC is able to directly account for the uncertainty of the weather forecast in its control decisions. It was also shown that the performance of SMPC depends on the quality of the weather prediction; SMPC performed clearly better using COSMO-7 predictions compared to the simple persistence predictions. A further benefit of SMPC is the easy tunability of the tradeoff between NRPE usage and comfort violations with the tuning parameter $\alpha$, which describes the level of constraint violations.

In summary, SMPC is a promising approach for building climate control. However, its performance in real applications can be expected to vary with the quality of the model and the available input data (model parameters, system states, weather predictions, etc.) to an extent that remains to be investigated.

\section{Acknowledgements}

Swisselectric Research, the Competence Center Energy and Mobility (CCEM-CH) and Siemens Building Technologies are gratefully acknowledged for their financial support of the OptiControl project.

\section{References}

[1] International Energy Agency, Energy Efficiency Requirements in Building Codes - Energy Efficiency Policies for New Buildings, 2008.

[2] M. Gwerder, J. Tödtli, Predictive control for integrated room automation, in: Proc. of Clima - RHEVA World Congress 2005, Lausanne, Switzerland, 2005.

[3] OptiControl Project, www.opticontrol.ethz.ch (accessed 31.08.11).

[4] R. Andersen, B. Olesen, J. Toftum, Simulation of the effects of occupant behaviour on indoor climate and energy consumption, in: Proc. of Clima RHEVA World Congress 2007, Helsinki, Finland, 2007.

[5] C.R.D. Bourgeois, I. Macdonald, Adding advanced behavioural models in whole building energy simulation: a study on the total energy impact of manual and automated lighting control, Energy and Buildings 38 (2006) 814-823.

[6] S. Cho, M. Zaheer-uddin, Predictive control of intermittently operated radiant floor heating systems, Energy and Conversion Management 44 (2003) 1333-1342.

[7] W. Grünenfelder, J. Tödtli, The use of weather predictions and dynamic programming in the control of solar domestic hot water systems, in: Proc. of Mediterranean Electrotechnical Conference (Melecon), Madrid, Spain, 1985.

[8] G. Henze, D. Kalz, S. Liu, C. Felsmann, Experimental analysis of model-based predictive optimal control for active and passive building thermal storage inventory, International Journal of HVAC \& Research 11 (2005) 189-214.

[9] OptiControl Website, www.opticontrol.ethz.ch/Literature.html (accessed 31.08.11).

[10] G. Henze, C. Felsmann, G. Knabe, Evaluation of optimal control for active and passive building thermal storage, International Journal of Thermal Sciences 43 (2004) 173-183.

[11] G. Henze, C. Felsmann, G. Knabe, Impact of forecasting accuracy on predictive optimal control of active and passive building thermal storage inventory, International Journal of HVAC \& Research 10 (2004) 153-178.

[12] Y. Ma, F. Borrelli, B. Hencey, A. Packard, S. Bortoff, Model predictive control of thermal energy storage in building cooling systems, in: Proc. of 48th IEEE Conference on Decision and Control and 28th Chinese Control Conference, Shanghai, China, 2009.

[13] J. Siroky, F. Oldewurtel, J. Cigler, S. Privara, Experimental analysis of model predictive control for an energy efficient building heating system, Applied Energy 88 (9) (2011) 3079-3087.

[14] J. Maciejowski, Predictive Control with Constraints, Prentice Hall, 2002.

[15] J. Rawlings, D. Mayne, Model Predictive Control: Theory and Design, Nob Hill Publishing, 2009.

[16] BSI, EN 15251:2007, Indoor Environmental Input Parameters for Design and Assessment of Energy Performance of Buildings Addressing Indoor Air Quality, Thermal Environment, Lighting and Acoustics.

[17] P. Goulart, E. Kerrigan, J. Maciejowski, Optimization over state feedback policies for robust control with constraints, Automatica 42 (2006) 523-533.

[18] F. Oldewurtel, C. Jones, M. Morari, A Tractable approximation of chance constrained stochastic MPC based on affine disturbance feedback, in: Proc. of 47th IEEE Conf. on Decision and Control, Cancun, Mexico, 2008, pp. 4731-4736.

[21] F. Oldewurtel, R. Gondhalekar, C. Jones, M. Morari, Blocking parameterizations for improving the computational tractability of affine disturbance feedback MPC Problems, in: Proc. of 48th IEEE Conference on Decision and Control and 28th Chinese Control Conference, Shanghai, China, 2009.

[20] A. Parisio, Handling Uncertainty with Application to Indoor Climate Control and Resource Allocation Planning, PhD Thesis, University of Sannio, Benevento, Italy, 2009. 
[21] F. Oldewurtel, A. Parisio, C. Jones, M. Morari, D. Gyalistras, M. Gwerder, V. Stauch, B. Lehmann, K. Wirth, Energy efficient building climate control using stochastic model predictive control and weather predictions, in: Proc. of American Control Conference, 2010.

[22] J. Steppeler, G. Doms, U. Schättler, H. Bitzer, A. Gassmann, U. Damrath, G. Gregoric, Meso-gamma scale forecasts using the non-hydrostatic model LM, Meteorology and Atmospheric Physics 82 (2003) 75-96.

[23] V. Stauch, F. Schubiger, P. Steiner, Local weather forecasts and observations, Tech. Rep., in [28], 2009.

[24] F. Anderson, J. Moore, Optimal Filtering, Prentice Hall, 1979.

[25] TRNSYS Simulation Software, http://sel.me.wisc.edu/trnsys/ (accessed 31.08.11).

[26] EnergyPlus Energy Simulation Software, http://apps1.eere.energy.gov/ buildings/energyplus/ (accessed 31.08.11)

[27] B. Lehmann, K. Wirth, S. Carl, V. Dorer, T. Frank, M. Gwerder, Modeling of buildings and building systems, Tech. Rep., in [28], 2009.

[28] D. Gyalistras, M. Gwerder (Eds.), Use of weather and occupancy forecasts for optimal building climate control (OptiControl): two years progress report,
Tech. Rep., ETH Zurich and Siemens Building Technologies Division, Siemens Switzerland Ltd., Zug, Switzerland, 2009.

[29] R. Griffith, R. Steward, A nonlinear programming technique for the optimization of continuous processing systems, Journal of Management Science 7 (1961) 379-392.

[30] A. Prékopa, Stochastic Programming, third ed., Kluwer Academic Publishers, Dordrecht, Netherlands, 1995.

[31] E. Kerrigan, Robust Constraint Satisfaction: Invariant Sets and Predictive Control, PhD Thesis, University of Cambridge, 2000.

[32] CPLEX Optimization Software, www-01.ibm.com/software/integration/ optimization/cplex-optimizer/ (accessed 31.08.11)

[33] D. Gyalistras, B. Lehmann, K. Wirth, M. Gwerder, F. Oldewurtel, V. Stauch, Performance bounds and potential assessment, Tech. Rep., in [28] 2009.

[34] D. Gyalistras, K. Wirth, B. Lehmann, Analysis of savings potentials and peak electricity demand, Tech. Rep., in [28], 2009.

[35] F. Oldewurtel, D. Gyalistras, C. Jones, A. Parisio, M. Morari, Analysis of model predictive control strategies, Tech. Rep., in [28], 2009. 